\title{
Beyond the Transnosographic Emphasis on Psychosis: Nosological Perspectives on Schizophrenia and Its Prevention
}

\author{
Anna Comparelli ${ }^{*}$, Andrea Raballo ${ }^{2}$, Maurizio Pompili ${ }^{3}$ and Silvana Galderisi ${ }^{4}$ \\ ${ }^{1}$ Department of Psychiatry, Sant'Andrea Hospital of Rome, Rome, Italy ${ }^{2}$ Division of Psychiatry, Clinical Psychology and \\ Rehabilitation, Department of Medicine, University of Perugia, Perugia, Italy ${ }^{3}$ Department of Neurosciences, Mental Health \\ and Sensory Organs, Suicide Prevention Center, Sant'Andrea Hospital, Sapienza University of Rome, Rome, Italy \\ ${ }^{4}$ Department of Psychiatry, University of Campania Luigi Vanvitelli, Naples, Italy
}

OPEN ACCESS

Edited by:

Christian Huber,

University Psychiatric Clinic

Basel, Switzerland

Reviewed by:

Philippe Conus,

Centre Hospitalier Universitaire

Vaudois, Switzerland

${ }^{*}$ Correspondence:

Anna Comparelli

anna.comparelli@uniroma1.it

Specialty section:

This article was submitted to

Schizophrenia,

a section of the journal

Frontiers in Psychiatry

Received: 12 July 2019

Accepted: 19 August 2019

Published: 18 September 2019

Citation:

Comparelli A, Raballo A, Pompili M and Galderisi S (2019) Beyond the

Transnosographic Emphasis on Psychosis: Nosological Perspectives on Schizophrenia and Its Prevention.

Front. Psychiatry 10:666.

doi: 10.3389/fpsyt.2019.00666
Keywords: schizophrenia, early psychosis, nosology, extended psychosis phenotype, psychosis spectrum

There is an ongoing debate in the literature regarding relative merits and weaknesses of the concept of transnosographic psychosis, namely the fact that psychosis is not limited to any specific psychotic disorder but rather represents a continuous expression across the spectrum of psychoses and may be perceived as an extreme expression of quantitative traits continuously distributed in the general population. The genesis of this concept is complex and partly derived from research on the early detection of psychosis. Even within the established categorical system, in the last edition of the DSM (1), the chapter "Schizophrenia Spectrum and Other Psychotic Disorders" aimed to capture the underlying dimensional structure of psychosis. At this point in the debate, while scholars discuss a change of name of schizophrenia, an overview of possible nosological perspectives for schizophrenia is a mandatory step, both for clinical practice and for research.

The notion of a broad psychotic spectrum was strongly advocated by Guloksuz and van Os (2), who provocatively announced the "slow death" of the concept of schizophrenia, redefined as the poor outcome fraction of a "truly complex, multidimensional psychotic syndrome." According to this view, in around $10-20 \%$ of the non-ill general population (3), subtle alterations in processing environmental stimuli during daily life give rise to momentary and fluctuating paranoid feelings, or negative affective states (microphenotype). Some of these states tend to persist over time and occur in the presence of other symptoms such as depression and anxiety, giving rise to noticeable experiences of reality distortion (extended phenotype). The copresence of affective dysregulation and reality distortion (psychotic experiences) increases the risk of onset of psychotic disorder (illness macrophenotype), in a sort of psychopathological network of symptoms causally impacting each other over time. In this view, schizophrenia represents a rare mental disorder with poor prognosis within a broader spectrum of psychotic disorders.

The extended psychosis phenotype is trans-nosographic in nature, implying that it is not restricted to any specific psychotic disorder but rather represents both a continuous expression across the psychosis spectrum and an extreme expression of continuously distributed quantitative traits in the general population.

This model should be compared with the paradigm of clinical high risk (CHR), which is conditionally meant to identify those at risk of psychosis among symptomatic, help-seeking subjects.

The CHR paradigm (4-6) was developed with the description of three possible prodromal syndromes-attenuated positive symptom syndrome (APS), genetic risk and deterioration (GRD) and/or brief intermittent psychotic syndrome (BIPS). APS requires the presence of at least one attenuated positive psychotic symptom (unusual thought content, suspiciousness, grandiose ideas, perceptual abnormalities, or disorganized communication) of insufficient severity to meet 
diagnostic criteria for a psychotic disorder. The attenuated psychotic symptom(s) had to have begun or worsened in the past year. The GRD requires a combination of both functional decline (at least a 30\% decrease in Global Assessment of Function score over the last month compared to 12 months ago) and genetic risk; genetic risk refers to having either schizotypal personality disorder or a first-degree relative with a schizophrenia spectrum disorder. The BIPS state requires the presence of any one or more threshold positive psychotic symptoms (unusual thought content, suspiciousness, grandiosity, perceptual abnormalities, and disorganized communication) that are self-limited and too brief (less than one week) to meet diagnostic criteria for a brief psychotic disorder.

The transition rate to psychosis from a CHR state is approximately $20 \%$ over 1 year and $36 \%$ within three years; among those who convert, about $60 \%$ of diagnostic outcomes develop towards the schizophrenia spectrum and the remaining $40 \%$ towards mood-related and atypical forms of psychosis (7). Notably, however, only a minority of patients who do not convert will remit (7\%), whereas most patients will remain in a comparable state of impairment with continuing levels of attenuated psychotic-like symptoms and functional impairment (30\%); others will develop another psychiatric disorder, which is not necessarily associated with psychosis (60\%) (8).

Thus, on the one hand the CHR model highlights the existence of a large prevalence of attenuated psychotic symptoms, while on the other it has also been shown to have poor predictive specificity for schizophrenia, since of those who can be classified as CHR, only about $10-15 \%$ evolve towards schizophrenia (roughly half of those who convert to full blown psychosis). This is mainly due to two reasons: 1) total exclusion from the CHR criteria of negative and most disorganized symptoms; 2) restriction of the realm of schizophrenia to its mainly delusional-hallucinatory overt forms (9).

Indeed, since the initial conceptualization of CHR status, it was clear that the severity of specific symptoms at baseline (bizarre thinking, social withdrawal, and reduced global functioning) was related to transition to schizophrenia $(4,10,11)$. Furthermore, consistent with basic symptom and self-experience disorder research, specific subjective disturbances were found to be predictive of transition to schizophrenia (12).

If the breadth of these symptoms were included in CHR criteria, they would presumably lead to a more accurate prediction of schizophrenia rather than of first psychotic episode in a broad, trans-nosographic sense; nevertheless, positive symptoms are the central pivot of prodromal syndromes and somehow orient the predictive power of the construct towards delusional-hallucinatory outcomes.

In conclusion, the original dual-output model (transition/ no transition), due to its constitutive dimensional structure, has been the breeding ground for the trans-nosographic concept of attenuated psychosis. Indeed, attenuated psychosis is best understood as an early subclinical stage, harbouring the potential for pluripotent, transdiagnostic outcome trajectories, including both psychotic and non-psychotic ones.

Intense scientific debate has taken place around these issues. The main point of discrimination is no longer whether CHR status is sufficiently predictive of schizophrenia, but whether it is specifically predictive of psychotic spectrum disorders or simply a risk condition for other non-psychotic psychiatric conditions $(13,14)$.

The drafting of the DSM-5 was strongly influenced by the pressing downsizing of the category towards a dimensional approach, to the point that schizophrenia is now part of a group of disorders belonging to a spectrum, sharing, at least on a phenotypic level, some of their characteristics. If we embrace a spectrum approach, a legitimate discussion emerges around the benefits of changing the name of schizophrenia, with the specific scope of going further than a mere semantic revision, but maintaining the original nucleus of the knowledge on schizophrenia.

At this point, it is useful to wonder if only the name should be changed, or the concept of schizophrenia itself. Our problem resembles the debate about psychiatric diseaeses as natural entities vs. descriptive diagnostic categories. Regarding this, Kendler (15) stated that "in our current project to study and justify the nature of psychiatric disorders, we should be largely pragmatic but not lose sight of a fundamental commitment, despite the difficulties associated with the reality of psychiatric illness." Reality is here referred to its philosophical meaning of natural entity.

In this light, in the redefinition of the name/concept/entity of schizophrenia, three possible perspectives may be outlined:

1) Schizophrenia as a stochastic result of pathogenetic paths based on persistent interplay of genes and the environment, and framed in the context of the transnosographic psychopathological network, which leads to poor outcomes of clinical dimensions (affective, positive, negative, and cognitive) that are overlapping and severely expressed. In this perspective, schizophrenia would basically denote the most extreme (and somehow residual) outcomes of severe mental illnesses.

2) Schizophrenia as the delusional-hallucinatory expression of the psychotic spectrum that, regardless of pathogenetic and outcome paths, is characterized by the expression of positive symptoms. Such a notion, which is a variant of einheitpsycoseunitary psychosis, and includes affective and non-affective clinical dimensions, resembles the more recent DSM orientation and may be extended to schizoaffective disorders as well as to to certain forms of mania.

3) Schizophrenia as a disease tending virtually to Jaspers' morbid entity, with its etiopathogenesis strongly linked to neurodevelopmental disorders akin to Kahlbaum-Hecker Hebephrenia, as recently overviewed (16). Predominant disorganized/cognitive and negative symptoms would characterize the illness early, even in its prodromal stages, which ought to be detected before, and regardless of the absence of frank-full blown positive psychotic symptoms, in order to avoid natural progression of the disease.

From the perspective of early detection and prevention, in the first case prevention of schizophrenia would imply a general model of universal prevention of severe psychopathology; in the second hypothesis, schizophrenia, being only one of the many disorders that can cause positive symptoms, is detectable through the current 
concept of CHR based on positive symptoms; in the third scenario, we might think of reformulating a prodromal model by referring to criteria including both cognitive/disorganized, blunted affect and social/academy withdrawal symptoms, and intermediate phenotypes (such as impaired social cognition and speed of processing). While the first two options might seem the smoothest and less problematic ones, the last option might be the most promising in terms of

\section{REFERENCES}

1. American Psychiatric Association. Diagnostic and statistical manual of mental disorders. US: American Psychiatric Press (2013). (fifth revision). doi: 10.1176/appi.books. 9780890425596

2. Guloksuz S, van Os J. The slow death of the concept of schizophrenia and the painful birth of the psychosis spectrum. Psychol Med (2018) 48:229-44. doi: $10.1017 /$ S0033291717001775

3. van Os J, Reininghaus U. Psychosis as a transdiagnostic and extended phenotype in the general population. World Psychiatry (2016) 15:118-24. doi: $10.1002 /$ wps. 20310

4. Cannon TD, Cadenhead K, Cornblatt B, Woods SW, Addington J, Walker E, et al. Prediction of psychosis in youth at high clinical risk: a multisite longitudinal study in North America. Arch Gen Psych (2008) 65:28-37. doi: 10.1001/archgenpsychiatry.2007.3

5. Woods SW, Addington J, Cadenhead KS, Cannon TD, Cornblatt BA, Heinssen R, et al. Validity of the prodromal risk syndrome for first psychosis: findings from the North American Prodrome Longitudinal Study. Schizoph Bull (2009) 35:894-908. doi: 10.1093/schbul/sbp027

6. McGlashan TH, Walsh BC, Woods SW. The Psychosis-Risk Syndrome. Handbook for Diagnosis and Follow-up. New York: Oxford University Press (2010)

7. Fusar-Poli P, Bonoldi I, Yung AR, Borgwardt S, Kempton MJ, Valmaggia L, et al. Predicting psychosis: meta-analysis of transition outcomes in individuals at high clinical risk. Arch Gen Psych (2012) 69:220-9. doi: 10.1001/archgenpsychiatry.2011.1472

8. Millan MJ, Andrieux A, Bartzokis G, Cadenhead K, Dazzan P, Fusar-Poli P, et al. Altering the course of schizophrenia: progress and perspectives. Nat. Rev. Drug Discov (2016) 15:485-515. doi: 10.1038/nrd.2016.28

9. Parnas J. A disappearing heritage: the clinical core of schizophrenia. Schizophr Bull (2011) 37:1121-30. doi: 10.1093/schbul/sbr081

10. Velthorst E, Nieman DH, Becker HE, van de Fliert R, Dingemans PM, Klaassen R, et al. Baseline differences in clinical symptomatology between improving scientific knowledge and offering the opportunity of better encompassing effective strategies for early identification.

\section{AUTHOR CONTRIBUTIONS}

All authors listed have made substantial, direct, and intellectual contribution to the work and approved it for publication.

ultra high risk subjects with and without a transition to psychosis. Schizoph Res (2009) 109:60-5. doi: 10.1016/j.schres.2009.02.002

11. Piskulic D, Addington J, Cadenhead KS, Cannon TD, Cornblatt BA, Heinssen R, et al. Negative symptoms in individuals at clinical high risk of psychosis. Psychiatry Res (2012) 30:220-4. doi: 10.1016/j. psychres.2012.02.018

12. Nelson B, Thompson A, Yung AR. Basic self-disturbance predicts psychosis onset in the ultra high risk for psychosis "prodromal" population. Schizophr Bull (2012) 38:1277-87. doi: 10.1093/schbul/sbs007

13. Woods SW, Powers AR, 3rd, Taylor JH, Davidson CA, Johannesen JK, Addington J, et al. Lack of diagnostic pluripotentiality in patients at clinical high risk for psychosis: specificity of comorbidity persistence and search for pluripotential subgroups. Schizoph Bull (2018) 15:254-63. doi: 10.1093/ schbul/sbx138

14. Fusar-Poli P. The hype cycle of the clinical high risk state for psychosis: the need of a refined approach. Schizophr Bull (2018) 44:250-3. doi: 10.1093/ schbul/sbx181

15. Kendler KS. The nature of psychiatric disorders. World Psychiatry (2016) 15:5-12. doi: 10.1002/wps.20292

16. Dalle Luche R, Maggini C. Genealogia della schizofrenia. In: Ebefrenia, dementia praecox, neurosviluppo. Mimesis (2018).

Conflict of Interest Statement: The authors declare that the research was conducted in the absence of any commercial or financial relationships that could be construed as a potential conflict of interest.

Copyright (c) 2019 Comparelli, Raballo, Pompili and Galderisi. This is an openaccess article distributed under the terms of the Creative Commons Attribution License (CC BY). The use, distribution or reproduction in other forums is permitted, provided the original author(s) and the copyright owner(s) are credited and that the original publication in this journal is cited, in accordance with accepted academic practice. No use, distribution or reproduction is permitted which does not comply with these terms. 